ISSN 0103-9954

\title{
OCORRÊNCIA DE ASSOCIAÇÃO MICORRÍZICA EM SEIS ESSÊNCIAS FLORESTAIS NATIVAS DO ESTADO DO RIO GRANDE DO SUL
}

\section{MYCORRHIZAL ASSOCIATION STUDIES IN SIX NATIVE FORESTRY SPECIES OF RIO GRANDE DO SUL STATE}

\author{
Robson Andreazza $^{1}$ Zaida Inês Antoniolli ${ }^{2}$ Vetúria Lopes de Oliveira ${ }^{3} \quad$ Lineu Trindade Leal $^{4}$ \\ Carlos Augusto Moro Junior ${ }^{4}$ Simone Pieniz ${ }^{5}$
}

\section{RESUMO}

Ao favorecer o crescimento das plantas hospedeiras, a micorriza pode ser um fator importante para as essências florestais nativas do estado do Rio Grande do Sul. O objetivo deste trabalho foi identificar o tipo de micorriza em seis espécies florestais do Estado: pinheiro-do-paraná (Araucaria angustifolia (Bertol.) Kuntze), timbaúva (Enterolobium contortisiliquum (Vell.) Morong), canafístula (Peltophorum dubium (Spreng) Taub), ipê-amarelo (Tabebuia chrysotricha (Mart. ex DC.) Standl.), ipê-roxo (Tabebuia heptaphylla (Well.) Toledo) e grápia (Apuleia leiocarpa (Vogel) J.F. Macbr). O estudo foi desenvolvido na Fepagro Floresta - Boca do Monte, Santa Maria, em bosques de espécies nativas e plantadas. As amostras de raízes, os corpos de frutificação dos fungos e o solo foram analisados no laboratório. As raízes foram processadas e analisadas quanto ao tipo de micorriza presente. Os fungos ectomicorrízicos nativos encontrados foram identificados, isolados e mantidos em cultura. As espécies estudadas não apresentaram colonização ectomicorrízica, embora em alguns locais tenham sido encontrados esporocarpos próximos às plantas. A associação com micorrizas arbusculares foi encontrada em todas as espécies de essências florestais nativas estudadas.

Palavras-chave: Peltophorum dubium; Tabebuia heptaphylla; ectomicorriza; endomicorriza.

\section{ABSTRACT}

Mycorrhizal associations could promote plant growth in native forestry species in Rio Grande do Sul State. The aim of this work was to identify mycorrhizal associations in six native forestry species: Araucaria angustifolia (Bertol.) Kuntze, Enterolobium contortisiliquum (Vell.) Morong, Peltophorum dubium (Spreng) Taub., Tabebuia chrysotricha (Mart. ex DC.) Standl., Tabebuia heptaphylla (Well.) Toledo) and Apuleia leiocarpa (Vogel) J.F. Macbr.). The study was done at Fepagro Forestry - Boca do Monte, Santa Maria, in cultivated and natural forest stands. Roots, fungal fruiting bodies and soil were analyzed in laboratory. Roots were processed and analyzed considering the formation of mycorrhizal association. Ectomycorrhizal fungi growing in the forest areas were identified, isolated and multiplied. The plants showed no ectomycorrhizal colonization, even though sporocarps of these fungi had been found close to the plants in some sites. The presence of arbuscular mycorrhizal was observed in all native forestry species studied.

Keywords: Peltophorum dubium; Tabebuia heptaphylla; ectomycorrhizae; endomycorrhizae.

\section{INTRODUÇÃO}

As florestas nativas são um ponto chave para a biodiversidade dos ecossistemas florestais. O estado do Rio Grande do Sul apresenta grande diversidade de espécies florestais nativas e com ampla abrangência em praticamente todo o território regional, mas o uso indiscriminado dos recursos naturais dessas áreas vem prejudicando o meio ambiente. Problemas ocasionados pelo desequilíbrio dos processos que sustentam os

1. Engenheiro Agrônomo, Doutorando do Programa de Pós-Graduação em Ciência do Solo, Universidade Federal do Rio Grande do Sul, Av. Bento Gonçalves, 7712, CEP 91540-000, Porto Alegre (RS). Bolsista CAPES. randreazza@mail.ufsm.br

2. Bióloga, Dra ${ }^{\mathrm{a}}$, Professora do Departamento de Solos, Centro de Ciências Rurais, Universidade Federal de Santa Maria, CEP 97105-900, Santa Maria (RS). zaida@smail.ufsm.br

3. Engenheira Agrônoma, Dr., Professora do Departamento de Microbiologia do Solo, Centro de Ciências Biológicas, Universidade Federal de Santa Catarina, Florianópolis (SC).

4. Acadêmicos do Curso de Agronomia, Universidade Federal de Santa Maria, Bolsistas CNPq e FIPE, CEP 97105900, Santa Maria (RS).

5. Acadêmica do Curso de Nutrição, Centro Universitário Franciscano (UNIFRA), Rua dos Andradas, 1614, CEP 97010-032, Santa Maria (RS).

Recebido para publicação em 25/06/2006 e aceito em 11/08/2008. 
ecossistemas, como perdas de produção em razão de inundações, esgotamento do solo e secas, fazem com que se tenha um panorama pouco otimista para as diferentes atividades econômicas no Estado (SCHROEDER, 1991).

O Estado possuía originalmente $42 \%$ de sua superfície territorial coberta com florestas, mas já no início do século XX, essa área foi reduzida para aproximadamente 25\%, chegando a apenas 4\% em 1965. Embora tenha ocorrido desmatamento, encontram-se pontos isolados de matas nativas e algumas estratégias de recuperação dessas florestas já foram propostas (ZACHIA et al., 2002). Essas matas estão localizadas na região sudoeste, região de Santa Maria e na metade norte do Rio Grande do Sul. As poucas áreas nativas existentes restringem-se às áreas de preservação, aos parques, às reservas e a pequenos porcentuais em áreas de difícil acesso. Essas áreas ocupam apenas pequena parte do sudoeste do Rio Grande do Sul. Segundo Reitz et al. (1983), o aspecto fitofisionômico é formado, sobretudo, pela predominância de algarrobo (Prosopis nigra) e inhanduvaí (P. algarobila).

No estudo da composição florística da região de Santa Maria, foram encontradas 58 espécies diferentes, com a família Myrtaceae sendo representada por quinze espécies, Leguminosae por cinco e Asteraceae por quatro. Os gêneros melhores representados foram Eugenia, com cinco espécies, Casearia, Dasyphyllum, Erythoxylum, Gomidesia e Sesbania, com duas espécies cada (ZÁCHIA et al., 2002).

Na região da Boca do Monte em Santa Maria, com uma grande diversidade florística, a família Myrtaceae é, também a mais característica da mata ciliar analisada, por apresentar maior número de gêneros (cinco) e espécies (sete). Destacam-se, ainda, as famílias Leguminosae com quatro gêneros e cinco espécies e Sapindaceae com três gêneros (CANTARELLI et al., 1999).

Carvalho (1998) indica outras espécies madeireiras nativas não-leguminosas do sul do Brasil como uma opção para o reflorestamento, como o pinheiro-do-paraná (Araucaria angustifolia), o pau-marfim (Balfourodendron riedelianum), a canjerana (Cabrela canjerana subsp. canjerana), pau-ferro (Myracrodruon balansae) e pessegueiro-bravo (Prunus brasiliensis). Já entre as espécies florestais nativas leguminosas, o autor cita o angico-vermelho (Anadenanthera colubrina var. cebil), angico-branco (Anadenanthera colubrina var. colubrina), angico-cascudo (Anadenanthera peregrina var. falcata), timbaúva (Enterolobium contortisiliquum), bracatinga (Mimosa scrabella), canafístula (Peltophorum dubium) e guapuruvu (Schizolobium parahyba).

Como se pode observar, a composição florística dessas formações está bem documentada, mas pouco se conhece sobre as associações com microorganismos nesses ecossistemas e suas interações com as plantas. Sabe-se que alguns organismos do solo podem auxiliar o estabelecimento de plantas em áreas de difícil adaptação, como áreas em processo de arenização. Dentre esses organismos, destacam-se os fungos micorrízicos que, ao associarem-se às raízes das plantas, desenvolvem estruturas eficientes na absorção de água e nutrientes que são, posteriormente, transferidos às plantas (SILVA, 2002; SILVA et al., 2003a; 2003b; 2003c; ANDREAZZA et al., 2003; 2004).

As micorrizas são associações simbióticas mutualísticas entre certos fungos do solo e a maioria das plantas vasculares. Por meio dessa associação, a planta fornece ao fungo carboidratos necessários ao seu crescimento, enquanto que o fungo, por meio de suas estruturas externas (hifas), auxilia na absorção dos nutrientes da solução do solo e os transfere para as plantas (PASCOAL e NAVARRO, 1985; ANTONIOLLI e KAMINSKI, 1991; SMITH e READ, 1997).

Dentre as micorrizas, as ectomicorrizas e as micorrizas arbusculares são aquelas com maior importância para as essências florestais. Há indícios de que as micorrizas arbusculares são importantes na fase inicial de desenvolvimento das espécies florestais. Embora a maioria dessas espécies seja colonizada pelos fungos ectomicorrízicos, na fase de muda, são as micorrizas arbusculares que parecem desempenhar um importante papel na absorção de nutrientes (ROJAS e SIQUEIRA, 2000; NOVAIS et al., 2005). Nesse sentido, têm sido observados efeitos benéficos de fungos micorrízicos arbusculares em plantas jovens. Caldeira et al. (1997) observaram maior taxa de sobrevivência de duas leguminosas arbóreas (Copaifera martii e Dimorphandra macrostachya) quando inoculadas com o fungo Gigaspora margarita. Bowen et al. (1974), observaram que, no estádio inicial de desenvolvimento de mudas de araucária, a colonização com endomicorrizas aumenta a absorção de zinco.

Entretanto, o crescimento e o estabelecimento de muitas plantas de interesse florestal são 
beneficiados pela colonização por fungos ectomicorrízicos. Isso resulta dos efeitos morfogenéticos proporcionados ao sistema radicular por esses fungos, como aumento da ramificação das raízes e redução da dominância apical (SMITH e READ, 1997; MARX e CORDELL, 1989). As ectomicorrizas promovem um incremento significativo da área de absorção radicular das plantas colonizadas, maximizando o aproveitamento de água e nutrientes, como o fósforo (P), o nitrogênio (N) e o potássio (K) (SMITH e READ, 1997; MARX e CORDELL, 1989). Acredita-se que a absorção de P é de duas a cinco vezes maiores nas raízes com ectomicorrizas do que nas raízes não-colonizadas. Além disso, propiciam melhor resistência ao estresse hídrico, a temperaturas elevadas, à acidez e maior tolerância às condições de toxidez do solo. Também atuam na defesa do sistema radicular contra patógenos (SMITH e READ, 1997). Com isso, contribuem para o estabelecimento e desenvolvimento das plantas, mesmo em solos com baixos teores de nutrientes ou degradados (MARX e CORDELL, 1989).

Assim, o conhecimento sobre a ocorrência das associações micorrízicas, em especial em áreas degradadas, poderá contribuir para melhor explorar os efeitos benéficos da simbiose entre esses fungos e as mudas das espécies florestais usadas no reflorestamento. O objetivo deste trabalho foi identificar os tipos e época de ocorrência de associação micorrízica em seis espécies florestais nativas do estado do Rio Grande do Sul.

\section{MATERIAL E MÉTODOS}

O estudo foi desenvolvido na Fepagro Florestas, Santa Maria, RS e no Departamento de Solos da Universidade Federal de Santa Maria (UFSM). Na Fepagro Florestas, foram realizadas as coletas de corpos de frutificação dos fungos ectomicorrízicos, raízes das essências florestais e solo. Posteriormente, o material foi analisado no Laboratório Prof. Marcos Rubens Fries para identificação, isolamento dos fungos ectomicorrízicos e avaliação morfológica das raízes das espécies florestais estudadas.

\section{Espécies nativas estudadas}

As espécies nativas estudadas foram: pinheiro-do-paraná (Araucaria angustifolia), timbaúva (Enterolobium contortisiliquum), canafístula (Peltophorum dubium), ipê-amarelo (Tabebuia chrysotricha), ipê-roxo (Tabebuia heptaphylla), e grápia (Apuleia leiocarpa). Essas espécies foram escolhidas com base em sua importância ecológica e econômica.

\section{Caracterização das áreas de coleta}

A coleta foi realizada na Fepagro Floresta, em área de aproximadamente 280 ha, localizada em Boca do Monte no município de Santa Maria, Rio Grande do Sul. Na área de araucária o terreno é plano, o bosque possui 10 ha, com plantas com idade de 40 anos, sendo localizado próximo ao plantio de pinus (Pinus elliottii). A área de mata nativa estudada estava localizada próximo à sede da Fepagro Floresta e apresenta camada de serrapilheira $(20 \mathrm{~cm})$ e pequenas palmeiras.

As áreas de canafístula, timbaúva, ipê-roxo e grápia estão localizadas próximas uma das outras e em terreno ondulado, sendo em ordem da parte mais alta (próximo à rodovia) até a parte mais baixa (indo próximo ao centro da estação), em declive. Essas quatro espécies foram plantadas na mesma época e têm 17 anos de implantação. A vegetação herbácea predominante nesta área é de gramíneas, com alta densidade e tamanho.

Em todas as áreas das espécies nativas pré-determinadas, foram encontradas plantas de pinus próximas ou dentro das áreas de coleta. As plantas foram marcadas com tinta branca, sendo selecionadas cinco plantas de cada espécie em cada área, escolhidas aleatoriamente e de forma a representar a população de plantas na área.

\section{Período de coleta}

A coleta a campo foi efetuada em três épocas do ano, no outono, inverno e primavera de 2004.

Na mata de araucária, a coleta foi realizada nos dias 13/05, 23/08 e 25/11/2004. Na de ipê- amarelo, as coletas foram feitas nos dias 24/06, 28/08 e 25/11/2004. E, nas de canafístula, timbaúva, ipê roxo e grápia amarela, as coletas ocorreram nos dias 20/05, 21/10 e 21/11/2004.

\section{Coletas de corpos de frutificação dos fungos ectomicorrízicos}

Após a marcação das árvores, foi realizada, dentro do perímetro da copa da planta, a contagem dos 
corpos de frutificação dos fungos ectomicorrízicos, e foi medida a distância entre esses corpos de frutificação e o tronco da planta. O local em que o corpo de frutificação se encontrava foi marcado com uma estaca de madeira e enumerado com o mesmo número do corpo de frutificação. Os corpos de frutificação foram colocados em sacos de papel e levados ao laboratório acondicionados em caixa de isopor.

\section{Isolamento e multiplicação}

Os corpos de frutificação foram catalogados e fotografados. Parte destes foi secada em estufa a $50^{\circ} \mathrm{C}$ e guardadas em local seco. Os corpos de frutificação restantes foram utilizados para o isolamento do fungo e obtenção de culturas puras, conforme descrito por Brundrett et al. (1996). Antes do isolamento, os corpos de frutificação foram desinfestados pincelando-se externamente com álcool $70 \%$. Essas estruturas foram manualmente quebradas ao meio, retirando-se uma pequena porção do tecido no interior e semeando-a em meio Hagem sólido modificado (MODESS, 1941, apud MOLINA e PALMER, 1982) em placa de Petri, seguindo-se de incubação a $25^{\circ} \mathrm{C}$, durante 3 dias, até o aparecimento de micélio. Após esse período, as culturas sem contaminação evidente, e nas quais se observou o aparecimento de micélio, foram transferidas para placas contendo meio de cultura sólido Melin-Norkrans Modificado (MNM) (MARX, 1969) e mantidas sob as mesmas condições de incubação.

\section{Coleta de raízes e solo}

As coletas das raízes e do solo foram efetuadas simultaneamente às coletas dos corpos de frutificação. O solo coletado foi homogeneizado e separado dos restos vegetais para a análise química no Laboratório Central de Análises de Solo da Universidade Federal de Santa Maria.

As raízes foram lavadas em água corrente dentro de uma peneira $50 \mu \mathrm{m}$ e armazenadas em $80 \mathrm{~mL}$ de álcool 50\% até sua observação ao microscópio. Após esse período, foram observadas sob microscópio estereoscópio para descrição da morfologia radicular e análise da colonização micorrízica. Para isso, as raízes foram cortadas em pedaços de $5 \mathrm{~cm}$, separando-se uma porção representativa de 30g, guardadas em álcool 50\% para posterior caracterização do tipo de associação micorrízica presente.

\section{Avaliações das raízes}

A avaliação da colonização micorrízica foi feita por meio de três procedimentos: a) pela identificação a olho nu e sob lupa das alterações morfológicas, possivelmente provocadas por fungos ectomicorrízicos; b) no Laboratório de Ectomicorrizas, Departamento de Microbiologia e Parasitologia da Universidade Federal de Santa Catarina (UFSC), Florianópolis, onde foram realizados cortes transversais nas raízes como auxílio de um criomicrótomo, para visualização em microscópio, procurando-se observar a existência de estruturas como o manto e rede de Hartig, características da associação ectomicorrízica (BRUNDRETT et al., 1996); c) as associações micorrízicas arbusculares foram observadas conforme metodologia de Brundrett et al. (1996).

\section{Identificação dos isolados de fungos ectomicorrízicos}

A identificação final dos isolados foi feita no Laboratório de Ectomicorrizas da UFSC e com o auxílio do sites INVAM (2004) e BCERN (2004), que possui fotos ilustrativas e descrição de caracteres taxonômicos, e morfológicos como cor, tamanho e forma, entre outras características dos fungos micorrizicos.

\section{RESULTADOS E DISCUSSÃO}

Não foram observadas associações ectomicorrízicas nas seis espécies florestais nativas estudadas. Entretanto, todas apresentaram associação com fungos micorrízicos arbusculares (Tabela 1). Zangaro et al. (2002) observaram que o ipê-roxo, ipê-amarelo e a timbaúva apresentavam micorrizas arbusculares, mas esses autores não detectaram a ocorrência dessa associação em canafístula. Andrade et al. (2000), estudando o status micorrízico de espécies nativas da Mata Atlântica e da Floresta de Araucária em Santa Catarina, detectaram somente micorrizas do tipo arbuscular nas 29 espécies examinadas, pertencentes a 19 famílias, sendo várias espécies de Myrtaceae e de Leguminosae.

Entretanto, muitas essências florestais nativas são potencialmente capazes de formar os dois tipos de associação micorrízica; micorrizas arbusculares e ectomicorrizas, como é o caso de algumas espécies das famílias Leguminosae e Myrtaceae (STÜRMER e BELLEI, 1994). Em levantamentos realizados em florestas 
de eucalipto em Santa Catarina, em solos arenosos, observou-se que a idade da planta tem influência sobre o tipo de associação micorrízica. Inicialmente há uma alta esporulação e colonização por fungos micorrízicos arbusculares (FMAs) e esta diminui com a idade da planta (BELLEI, 1987). Espécies importantes da subfamília Caesalpinioideae são naturalmente colonizadas por fungos ectomicorrízicos (SMITH e READ, 1997), enquanto que espécies arbóreas da subfamília Papilionoideae estão associadas normalmente a FMAs. Já, algumas espécies da subfamília Mimosoideae podem associar-se tanto com fungos ectomicorrízicos quanto com fungos micorrízicos arbusculares (GROVE e LE TACON, 1993).

Além da questão de compatibilidade fungo-hospedeiro, outros fatores biológicos podem também estar relacionados à ocorrência de propágulos de um ou outro tipo de micorriza em determinados locais como a qualidade e o nível do inóculo micorrízico presente no local (AMARANTHUS e PERRY, 1994; OLIVEIRA et al., 1997; DODD e THOMSON, 1994). Ocorre um declínio do potencial de colonização em solos onde as plantas nativas foram removidas e onde plantas exóticas foram introduzidas (AMARANTHUS e PERRY, 1994; OLIVEIRA et al., 1997).

Dessa forma, é possível que as espécies examinadas, embora possam ser capazes de se associar a fungos ectomicorrízicos, não tenham encontrado inóculo desses fungos nos locais estudados. Entretanto, a existência nas proximidades de hospedeiros comprovados de fungos ectomicorrízicos como é o caso de Pinus spp., juntamente com a presença de corpos de frutificação de fungos ectomicorrízicos, parece contradizer essa hipótese. Assim, trabalhos futuros devem considerar outras regiões, solo, idade das plantas e condições climáticas.

A ocorrência de micorrizas arbusculares em Araucaria angustifolia já foi relatada por vários autores (OLIVEIRA et al., 1992; McGEE et al., 1999; ANDRADE et al., 2000) não havendo, entretanto, relatos de ocorrência de ectomicorrizas nessa espécie (MUCHOVEJ et al., 1992). Além disso, alguns estudos demonstraram o efeito benéfico desse tipo de associação sobre o crescimento dessa espécie (MUCHOVEJ et al., 1992; OLIVEIRA et al., 1992; ZANDAVALLI et al., 2004), evidenciando a importância das micorrizas arbusculares para essa essência florestal.

TABELA 1: Presença (+) ou ausência (-) de colonização micorrízica arbuscular (AM) e ectomicorrízica (ECM) em seis espécies florestais nativas do estado do Rio Grande do Sul, 2006.

TABLE 1: Presence (+) or absence (-) of arbuscular mycorrhizal colonization (AM) and ectomycorrhizal (ECM) colonization in six native forestry species from State of Rio Grande do Sul, 2006.

\begin{tabular}{|c|c|c|c|}
\hline Nome comum & Nome científico & AM & ECM \\
\hline Pinheiro-do-paraná & Araucaria angustifolia & + & - \\
\hline Timbaúva & Enterolobium contortisiliquum & + & - \\
\hline Canafístula & Peltophorum dubium & + & - \\
\hline Ipê-amarelo & Tabebuia chrysotricha & + & - \\
\hline Ipê-roxo & Tabebuia heptaphylla & + & - \\
\hline Grápia & Apuleia Ieiocarpa & + & - \\
\hline
\end{tabular}

Araucárias de florestas nativas podem formar associações endomicorrízicas com maior porcentagem de colonização que em áreas de reflorestamento, e sua colonização pode ficar na faixa de 20 a $40 \%$ (MOREIRA et al., 2005). Outros autores salientam que a araucária é uma planta micotrófica e forma associação com fungos micorrízicos arbusculares (CARDOSO et al., 2005).

De acordo com levantamentos sobre micorrizas, realizados em florestas de Araucaria angustifolia e de Cabralea cangerana no sul do Brasil na Mata Atlântica, observou-se ocorrência somente de fungos arbusculares (ANDRADE et al., 2000). Em outro estudo, em florestas nativas no Uruguai, a espécie Peltophorum dubium teve uma colonização com fungos micorrízicos arbusculares de $48 \%$, não sendo encontrada colonização ectomicorrízica e Enterolobium sp. não apresentou qualquer tipo de colonização (FRIONI et al., 1999).

Em relação à época de coleta, não foram observadas variações na colonização micorrízica arbuscular (Tabela 2). Isso também foi observado por Novais et al. (2005) ao estudar a influência sazonal sobre a colonização de Brachiaria decumbens por fungos micorrízicos arbusculares.

A presença de corpos de frutificação de fungos ectomicorrízicos parece estar relacionada à presença de plantas de pinus nas imediações dos locais de coleta. As espécies desse gênero são hospedeiros comprovados 
de fungos ectomicorrízicos. Foram encontrados corpos de frutificação de fungos ectomicorrízicos somente no outono, evidenciando melhores condições ambientais, como temperatura e umidade para o desenvolvimento dessas estruturas (Tabela 2). Resultados semelhantes foram obtidos por Giachini et al. (2000 e 2004) ao estudar a ocorrência de fungos ectomicorrízicos em plantações de Eucalyptus spp. e Pinus spp. em Santa Catarina. Nessas plantações, a quantidade de corpos de frutificação foi maior no outono e no inverno. Assim, a associação com fungos arbusculares em espécies florestais pode indicar uma relação importante para o benefício da planta. Entretanto, mais estudos, nessa área, são necessários para caracterizar a função e importância dos fungos micorrízicos (ANDREAZZA, 2006). Os fungos ectomicorrizicos ocorrem naturalmente em importantes espécies florestais em quase todas as regiões da terra. Entre as gimnospermas, todos os representantes da família Pinaceae, ou seja, os gêneros Pinus, Pinacea, Abies, Laris, Tsuga e Pseudotsuga são ectomicorrízicos (MEYER, 1973). Dentre as angiospermas se pode citar os gêneros Salix, Populus, Carya, Eucalyptus, Fagus e Quercus. Estima-se que 2.100 espécies de fungos micorrízicos se associam com essências florestais nos EUA, sendo a maioria composta pelos basidiomicetos e alguns ascomicetos (MARX, 1975).

Considerando a predominância de associações micorrízicas do tipo arbuscular nas espécies nativas e o fato de que as micorrizas podem alterar o crescimento, sobrevivência e habilidade competitiva das espécies florestais, trabalhos futuros devem contemplar o levantamento dos fungos e o estudo da dependência micorrízica das espécies nativas em suas várias fases de desenvolvimento.

TABELA 2: Fungos ectomicorrízicos em três épocas do ano em povoamentos de seis espécies florestais nativas do estado do Rio Grande do Sul, 2006.

TABLE 2: Ectomycorrhizal fungi in tree different periods of the year in six native forest from State of Rio Grande do Sul, 2006.

\begin{tabular}{|c|c|c|c|c|}
\hline Nome comum & Época de coleta & n. esporocarpos & Fungos* & $\mathrm{DP}(\mathrm{cm})^{* *}$ \\
\hline \multirow{3}{*}{ Pinheiro-do-paraná } & Outono & 48 & Laccaria sp. & 164 \\
\hline & Inverno & - & - & - \\
\hline & Primavera & - & - & - \\
\hline \multirow{3}{*}{ Timbaúva } & Outono & - & - & - \\
\hline & Inverno & - & - & - \\
\hline & Primavera & - & - & - \\
\hline \multirow{3}{*}{ Canafístula } & Outono & - & - & - \\
\hline & Inverno & - & - & - \\
\hline & Primavera & - & - & - \\
\hline \multirow{4}{*}{ Ipê-amarelo } & Outono & 2 & Suillus sp. & 10 \\
\hline & & 2 & Scleroderma sp. & 40 \\
\hline & Inverno & - & - & - \\
\hline & Primavera & - & - & - \\
\hline \multirow{3}{*}{ Ipê-roxo } & Outono & - & - & - \\
\hline & Inverno & - & - & - \\
\hline & Primavera & - & - & - \\
\hline \multirow{3}{*}{ Grápia } & Outono & 9 & Lactarius sp. & 177 \\
\hline & Inverno & - & - & - \\
\hline & Primavera & - & - & - \\
\hline
\end{tabular}

Em que: *Fungos ectomicorrízicos encontrados próximos às espécies florestais; **Distância média entre o fungo ectomicorrízico e o tronco da planta; DP = distância da planta.

\section{CONCLUSÕES}

As espécies florestais nativas: araucária (Araucaria angustifolia), timbaúva (Enterolobium contortisiliquum), canafístula (Peltophorum dubium), ipê-amarelo (Tabebuia chrysotricha), ipê-roxo (Tabebuia heptaphylla), e grápia (Apuleia leiocarpa) apresentam associação com fungos micorrízicos arbusculares em seu habitat natural na Fepagro Florestas, Boca do Monte, no Rio Grande do Sul. 


\section{AGRADECIMENTOS}

À FEPAGRO Floresta (Fundação Estadual de Pesquisa Agropecuária) de Santa Maria, RS, pela ajuda e fornecimento da área para a realização da pesquisa; ao Sr. Paulo Pedrollo funcionário da Fepagro, pela ajuda e auxílio técnico no campo; ao Antônio Carlos Bassaco, Laboratorista do Departamento de Solos, pela ajuda nas coletas; ao Pedro Bonnassis e Luiz Borges (Laboratório de Ectomicorrizas/UFSC, Florianópolis, SC) pela orientação e ajuda na identificação e corte das raízes.

\section{REFERÊNCIAS BIBLOGRÁFICAS}

AMARANTHUS, M. P.; PERRY, D. A. The function of ectomycorrhizal fungi in the field: linkages in space and time. Plant and Soil, v. 159, n. 1, p. 133-140, 1994.

ANDRADE, A. C. S. et al. Mycorrhizal status of some plants of the Araucaria Forest and the Atlantic Rainforest in Santa Catarina, Brazil. Mycorrhiza, Heidelberg, v. 10, n. 3, p. 131-136, 2000.

ANDREAZZA, R. Associação de fungos ectomicorrízicos com espécies florestais nativas do Estado do Rio Grande do Sul. 2006. 73 f. Dissertação (Mestrado em Ciência do Solo) - Universidade Federal de Santa Maria, Santa Maria.

ANDREAZZA, R. et al. Espécies de Pisolithus sp. na produção de mudas de Eucaliptus grandis Hill ex Maiden em solo arenoso. Ciência Florestal, v. 14, n. 2, p. 51-59, 2004.

ANDREAZZA, R. et al. Avaliação de mudas de eucalipto inoculadas com fungos ectomicorrízicos em solo arenoso. In: CONGRESSO BRASILEIRO DE CIÊNCIA DO SOLO, 29., 2003, Ribeirão Preto. Anais... Ribeirão Preto: UESP, 2003.1 CD-ROM.

ANTONIOLLI, Z. I.; KAMINSKI, J. Micorrizas. Ciência Rural, v. 2, n. 3, p. 441-455, 1991.

BELLEI, M. Micorrizas de Eucalyptus spp. Em viveiros e florestas de Santa Catarina. Florianópolis: UFSC. 1987. $54 \mathrm{p}$.

BCERN - BRITSH COLUMBIA ECTOMYCORRHIZAL RESEARCH NETWORK. Disponível em: www.pcf.forestry.ca/biodiversity/bcern/index_e.html, Acesso em: 10 mai. 2004.

BOWEN, G. D.; SKINNER, M. F.; BEVEGE, D. I. Zinc uptake by mycorrhizal and uninfected roots of Pinus radiata and Araucaria cunninghamii. Soil Biology and Biochemistry, v. 6, n. 3, p. 141-144, 1974.

BRUNDRETT, M. et al. Working with mycorrhizas in forestry and agriculture. Canberra: ACIAR, 1996. 400 p.

CALDEIRA, M. V. W. et al. Crescimento de leguminosas arbóreas em resposta a inoculação com fungos micorrízicos arbusculares. Ciência Florestal, v. 7, n. 1, p. 1-10, 1997.

CANTARELLI, E. B. et al. Aspectos florísticos da floresta estacional decidual, às margens do Rio Ibicui-Mirim no distrito de Boca do Monte, Santa Maria-RS. In: CICLO DE ATUALIZAÇÃO FLORESTAL DO CONE-SUL, 1999, Santa Maria. Anais... Santa Maria: UFSM, 1999. p.255-261.

CARDOSO, E. J. B. N. et al. Fungos micorrízicos arbusculares em mata nativa e reflorestamento de Araucaria angustifolia impactado. In: CONGRESSO BRASILEIRO DE CIÊNCIA DO SOLO. 30., 2005, Recife. Anais... Recife: UFPE, 2005. 1 CD-ROM.

CARVALHO, P. E. R. Espécies alternativas para o reflorestamento e o seu futuro industrial nos estados do Sul do Brasil. Anais... In: SIMPÓSIO FLORESTAL DO RIO GRANDE DO SUL, 5.; SIMADER - RS, 1., 1998, Santa Maria - RS. Anais... Santa Maria, 1998. p.21-28.

DODD, J. C.; THOMSON, B. D. The screening and selection of inoculant of arbuscular mycorrhizal and ectomycorrhizal fungi. Plant and Soil, v. 159, n. 1, p. 149-158, 1994.

FRIONI, L.; MINASIAN, H.; VOLFOVICZ, R. Arbuscular mycorrhizae and ectomycorrhizae in native tree legumes in Uruguay. Forest Ecology and Management, v. 115, p. 41-47, 1999.

GIACHINI, A. J. et al. Ectomycorrhizal fungi in Eucalyptus and Pinus plantations in southern Brazil. Mycologia, v. 92, n. 6, p. 1166-1177, 2000.

GIACHINI, A. J.; SOUZA, L. A. B.; OLIVEIRA, V. L. Species richness and seasonal abundance of ectomycorrhizal fungi in plantations of Eucalyptus dunnii and Pinus taeda in southern Brazil. Mycorrhiza, v. 14, p. 375-381, 2004.

GROVE, T. S.; LE TACON, F. Mycorrhizal associations in plantation forestry. Advances in Plant Pathology, n. 23, v. 9, p.191-227, 1993.

INVAM , INTERNATIONAL CULTURE COLLECTION OF VESICULAR ARBUSCULAR MYCORRHIZAL FUNGI. Disponível http://invam.caf.wvu.edu/mycinfo/methods/cultures/monosp.htm. 20 de maio 2004.

MARX, D. H. The influence of ectotrophic mycorrhizal fungi on the resistance of pine roots to pathogenic infections. I. Antagonism of mycorrhizal fungi to root pathogenic fungi and soil bacteria. Phytopathology, v. 59, p. 153-163, 
1969.

MARX, D.H. - Mycorrhizae and stablishment of trees on strip mined land. The Ohio Journal of Science, v. 75, p. 289-97, 1975.

MARX, D. H.; CORDELL, C. E. The use of specific ectomycorrhizas to improve artificial forestation practices. In: WHIPPS, J. M.; LUMSDEN, R. D. (eds.). Biotechnology of fungi for improving plant growth. New York: 1989. p.1-25.

MEYER, F. H. - Distribution of ectomycorrhizae in native and man-made forest. In: MARKS, G. C.; KOZLOWSKI, T. T. (ed..) Ectomycorrhizae: their ecology and physiology. New York: Academic Press, 1973. p.79-105.

McGEE, P. A.; BULLOCK, S.; SUMMERELL, B. A. Structure of mycorrhizae of the Wollemi pine (Wollemia nobilis) and related Araucariaceae. Australian Journal of Botany, v. 47, p. 85-95, 1999.

MOLINA, R.; PALMER, J. G. Isolation, maintenance, and pure culture manipulation of ectomycorrhizal fungi. In: SCHENCK, N. C. (ed.). Methods and principles of mycorrhizal research. St. Paul: 1982. p.115-129.

MOREIRA, M. et al. Identificação de fungos micorrízicos arbusculares (FMAS) em raízes de Araucaria angustifolia através de métodos moleculares. In: CONGRESSO BRASILEIRO DE CIÊNCIA DO SOLO, 30. 2005, Recife, Anais... Recife: UFPE, 2005. 1 CD-ROM.

MUCHOVEJ, R. M. C. et al. Influência da inoculação com fungos ectomicorrízicos e MVA sobre o comportamento de mudas de Araucaria angustifolia (Bert.) O. Ktze. Hoehnea, v. 19, p. 9-18, 1992.

NOVAIS, C. B.; et al. Influência sazonal da época do estabelecimento das culturas de fungos micorrízicos arbusculares sobre a densidade de esporos. In: CONGRESSO BRASILEIRO DE CIÊNCIA DO SOLO, 30, 2005, Recife. Anais... Recife: UFPE, 2005. 1 CD-ROM.

OLIVEIRA, I. B.; et al. Eficiência de inóculo com micorrizas nativas em Araucaria angustifolia. Agropecuária Catarinense, v. 5, n. 4, p. 51-52, 1992.

OLIVEIRA, V. L.; SCHMIDT, V. D. B.; BELLEI, M. M. Patterns of arbuscular- and ecto- mycorrhizal colonization of Eucalyptus dunnii in southern Brazil. Annuaire des Sciences Forestières, v. 54, p. 73-481, 1997.

PASCOAL, J. O.; NAVARRO, J. M. B. Significado de los microorganismos del suelo em nutrición vegetal: Simbiosis Rhizobium leguminos y micorrizas VA. In: GARRIDO, M. L.; OROSTICA, C. G. (eds.) Nutrición Vegetal: algunos aspectos químicos y biológicos. Granada: Consejo superior de investigaciones científicas, 1985. p.151-196.

REITZ, R.; KLEIN, M.; REIS, A. Projeto Madeira do Rio Grande do Sul. Itajaí: Herbário Barbosa Rodrigues, 1983. ROJAS, E. P.; SIQUEIRA, J. O. Micorriza arbuscular e fertilização do solo no desenvolvimento pós-transplante de mudas de sete espécies florestais. Pesquisa Agropecuária Brasileira, v. 35, n.1, p.103-114, 2000.

SCHROEDER, M. Cobertura florestal do Rio Grande do Sul: Tendências e Perspectivas. In: SEMINÁRIO SOBRE A SITUAÇÃO FLORESTAL DO RIO GRANDE DO SUL, 1., 1991, Santa Maria. Anais... Santa Maria: 1991. p.01-09.

SILVA, R. F. População de fungos micorrízicos e influência de ectomicorrizas na produção de mudas de Eucalyptus grandis e Pinus elliottii em solo arenoso. 2002. 105 f. Dissertação (Mestrado em Ciência do Solo) Universidade Federal de Santa Maria, Santa Maria.

SILVA, R. F; ANTONIOLLI, Z. I; ANDREAZZA, R. Efeito da inoculação com fungos ectomicorrízicos na produção de mudas de Eucalyptus grandis Hill ex. Maiden em solo arenoso. Ciência Florestal, v. 13, n. 1, p. 33-42, $2003 a$.

SILVA, R. F. et al. Fungos ectomicorrízicos no desenvolvimento de mudas de Eucalyptus grandis Hill ex. Maiden. Bioscience Journal, v. 19, n. 3, p. 9-17, 2003b.

SILVA, R. F; ANTONIOLLI, Z. I; ANDREAZZA, R. Produção de mudas de Pinus elliottii Engelm. micorrizadas em solo arenoso. Ciência Florestal, v. 13, n. 2, p. 57-65, 2003c.

SMITH, S. E.; READ, D. J. Mycorrhizal symbiosis. London: Academic Press, 1997. 605 p.

STÜRMER, S. L.; BELLEI, M. M. Composition and seasonal variation of spore populations of Arbuscular Mycorrhizal fungi in dune soils in island of Santa Catarina, Brazil. Canadian Journal of Botany, v. 72, p. 359-365, 1994.

ZACHIA, R. A. et al. Florística e síndromes de dispersão de espécies lenhosas em um fragmento de floresta ripária em Santa Maria, RS. 2002. In: CICLO DE ATUALIZAÇÃO FLORESTAL DO CONE-SUL. A Floresta e o Meio Ambiente, 2., 2002, Santa Maria, Anais... Santa Maria: 2002, p.121.

ZANDAVAlLI, R. B.; DILlENBURG, L. R.; SOUZA, P. V. D. Growth response of Araucaria angustifolia (Araucariaceae) to inoculation with the mycorrhizal fungus Glomus clarum. Applied Soil Ecology, v. 25, p. 245-255, 2004.

ZANGARO, W. et al. Micorriza arbuscular em espécies arbóreas nativas da bacia do Rio Iibagi, Paraná. Cerne, v. 8, n. 1, p. 77-87, 2002. 